\title{
IMPORTÂNCIA DOS ASPECTOS SOCIOCULTURAIS NA GESTÃO DE EQUIPES EM AMBIENTES DE DESENVOLVIMENTO DISTRIBUÍDO DE SOFTWARE
}

\author{
Ariel Gustavo Zuquello ${ }^{1}$ \\ Rômulo de Aguiar Beninca ${ }^{2}$ \\ Yoji Massago ${ }^{3}$ \\ Elisa Hatsue Moriya Huzita ${ }^{4}$ \\ Edson OliveiraJr ${ }^{5}$
}

\begin{abstract}
RESUMO
Desenvolvimento distribuído de software (DDS) é uma forma de envolver diversas pessoas no processo de desenvolvimento de software, o qual pode ocorrer de várias formas, seja em grupos de desenvolvimento no próprio local de trabalho, em outras cidades, ou até em outros países. No entanto, diversos fatores são considerados para que essa distribuição de tarefas seja distribuída de forma eficaz. Essa eficácia é mensurada por fatores como: redução de custos com comunicação entre os membros dispersos fisicamente, sucesso na realização do projeto, satisfação entre os membros, dentre outros. O objetivo deste artigo é analisar de forma empírica em um domínio específico, como gestores de projetos reagem quando se deparam com uma situação de formação de equipes para trabalhar em ambientes de DDS. Para tal, foi realizada uma pesquisa com 30 participantes, onde foram analisados alguns aspectos por nível de relevância, as prioridades socioculturais (língua, comportamento e formação) e dispersão geográfica usada pelos participantes para distribuição das equipes em ambientes de DDS. Como conclusão, observa-se que o principal fator considerado para a criação de uma equipe para trabalhar em ambientes de DDS é o fator sociocultural língua, cuja evidência é corroborada com um fator de $\mathbf{0 . 8 1}$ na escala de correlação de Spearman.
\end{abstract}

Palavras-chave: Desenvolvimento distribuído de software. Estudo empírico. Gestão de equipes.

\section{IMPORTANCE OF SOCIOCULTURAL ASPECTS IN MANAGEMENT TEAMS IN ENVIRONMENTS OF DISTRIBUTED SOFTWARE DEVELOPMENT}

\footnotetext{
ABSTRACT

1 Mestrando em Ciência da Computação do Departamento de Informática (DIN) da Universidade Estadual de Maringá (UEM). E-mail: arielzuquello@gmail.com.

2 Mestrando em Ciência da Computação do Departamento de Informática (DIN) da UEM. E-mail: romulo.beninca@ifsc.edu.br.

3 Mestrando em Ciência da Computação do Departamento de Informática (DIN) da UEM. E-mail: yojimassago@gmail.com.

4 Doutorado em Engenharia Elétrica pela Universidade de São Paulo (USP) e atualmente é professora titular na UEM. E-mail: elisa.h.m.huzita@gmail.com.

5 Doutorado em Ciências de Computação e Matemática Computacional pela Universidade de São Paulo (USP) e atualmente é professor adjunto na UEM. E-mail: edson@din.uem.br.
}

ForSci.: r. cient. IFMG campus Formiga, Formiga, v. 3, n. 1, p. 01-17, jan./jun. 2015. 
Distributed software development (DSD) is a way to involve many people in software development, which can occur in many forms process is in development groups in the workplace, in other cities or even other countries. However, several factors are considered for this distribution of tasks is distributed effectively. This efficiency is measured by factors such as: cost reduction with communication between physically dispersed members, successful completion of the project, satisfaction among members, among others. The objective of this paper is to analyze empirically in a specific domain, such as project managers react when faced with a situation of teaming to work in environments DDS. To this end, a survey of 30 participants, where some areas were examined by level of importance, sociocultural priorities (language, behavior and training) and geographical dispersion used by participants for distribution teams in DDS environments was performed. In conclusion, it is observed that the main factor considered for the creation of a team to work in environments DDS is the sociocultural factor language, whose evidence is corroborated by a factor of 0.81 on the scale of Spearman correlation.

Keywords: Distributed software developmen. Empirical study. Management teams.

\section{INTRODUÇÃO}

Atualmente o software tem se tornado um componente estratégico para diversas áreas de negócio. Especialmente na área de Engenharia de Software (ES), mercados nacionais têm se transformado em mercados globais, criando novas formas de cooperação e competição que vão além das fronteiras dos países (HERBSLEB et al., 2001). Tem se tornado cada vez mais custoso e menos competitivo desenvolver softwares no mesmo espaço físico, na mesma organização ou até mesmo no mesmo país (PRIKLADNICKI, 2011).

Mesmo que a ES ainda não seja uma disciplina madura (KAROLAK, 1998), as melhorias nas ferramentas e métodos nas últimas décadas têm permitido que grupos distribuídos, com diferentes expectativas, possam formar uma equipe para trabalhar em um mesmo projeto geograficamente distribuídos (AUDY; PRIKLADNICKI, 2007). Assim objetivando a redução de custos com maior qualidade no processo de desenvolvimento, muitas empresas iniciaram investimentos nessa área.

Alguns fatores contribuíram para a aceleração do surgimento do DDS, dentre eles destacamos (HERBSLEB et al., 2001) (CARMEL; TIJA, 2005): (i) A necessidade de recursos globais para serem utilizados a qualquer hora; (ii) A vantagem de estar perto do mercado local, incluindo o conhecimento dos clientes e as condições locais para explorar as oportunidades de mercado; (iii) Pressão por time-to-market (tempo entre análise de um produto e sua disponibilização para a venda) utilizando as vantagens proporcionadas pelo fuso horário diferente, no desenvolvimento conhecido como follow-the-sun (24h contínuas, contando com as equipes fisicamente distantes). 
Algumas características diferem a DDS do desenvolvimento de software tradicional, destacamos as três citadas por Carmel (1999): distância física, diferença de fuso horário, diferenças socioculturais (idioma, tradições, costumes, normas e comportamentos).

O objetivo de um DDS é simples: obter vantagens competitivas organizacionais relacionadas a custo, qualidade e flexibilidade no desenvolvimento de software, buscando aumento de produtividade e minimização de riscos (HUZITA et al., 2008). Segundo a International Data Group (IDC) (2006), em média, pode-se ter uma economia entre $25 \%$ e $50 \%$ em termos de custo quando grandes projetos são transferidos para operações em outros países (offshore).

O objetivo deste trabalho consiste em conceituar, estudar aspectos, problemas encontrados e oportunidades para futuras pesquisas em DDS. Também fazer um estudo empírico, por meio de experimento, sobre um domínio regional de pessoas, para mensurar os principais aspectos socioculturais e físicos para seleção de membros para trabalhar como equipes em ambientes de DDS.

Este trabalho está estruturado da seguinte forma: a Seção 2 apresenta-se o Referencial Teórico, onde serão explicados aspectos conceituais. Já na Seção 3, é apresentado o Cenário de Estudo, aonde é descrito o cenário na qual está inserido o presente experimento. Na Seção 4, encontra-se o Estudo Experimental, onde será detalhado o experimento realizado para a escrita deste artigo: o material utilizado, os métodos de verificação utilizados, bem como os resultados obtidos, entre outras informações. Na seção 5, os Resultados e Discussões, serão discutidos, brevemente, os resultados obtidos no experimento. Por fim, na Seção 6 tem-se a Conclusão repassando informações sobre o resultado do experimento e perspectivas futuras pesquisas.

\section{REFERENCIAL TEÓRICO}

A globalização, o crescimento da importância dos sistemas de informação nas empresas e os processos de terceirização têm proporcionado o Desenvolvimento Distribuído de Software (DDS) (AUDY; PRIKLADNICKI, 2008).

O DDS tem sido caracterizado principalmente pela colaboração e cooperação entre departamentos de organizações e pela criação de grupos de pessoas que trabalham em conjunto, mas estão localizados em cidades ou países diferentes, distantes temporal e fisicamente. (PRIKLADNICKI et al., 2004, p. 44).

Em outras palavras, DDS é o desenvolvimento de um software por um grupo de pessoas que estão trabalhando em locais geograficamente dispersos. Esta dispersão pode ser ForSci.: r. cient. IFMG campus Formiga, Formiga, v. 3, n. 1, p. 01-17, jan./jun. 2015. 
tanto dentro de uma mesma cidade, mesmo país, ou até mesmo entre diferentes países. Este último caso, de dispersão internacional, é uma instância do DDS, chamada de Desenvolvimento Global de Software (Global Software Development (GSD)) (BORGES et. al. 2013).

Segundo Audy e Prikladnicki (2007), podem-se definir algumas razões que levam as empresas à utilização do DDS, dentre elas destacam-se: Demanda e Custos; Time-to-Market; Mercado e presença global; Rigor e experiência no desenvolvimento; Sinergia cultural: Escala.

A adoção de DDS pode resultar em vários benefícios para a organização. Porém, para se obter estes benefícios, se faz necessária a resolução de diversos problemas oriundos da dispersão dos membros da equipe (PRIKLADNICKI, 2003). O fato de trabalhar com uma equipe que tem os seus membros em locais diferentes pode acarretar vários outros problemas, além daqueles existentes em um desenvolvimento centralizado. A Tabela 1 exibe os principais desafios do DDS, segundo Audy e Prikladnicki (2007).

Tabela 1 - Principais desafios do DDS

\begin{tabular}{|l|l|}
\hline CATEGORIAS & DESAFIOS \\
\hline Pessoas & Confiança \\
& Conflitos \\
& Diferenças socioculturais \\
& Ensino de DDS \\
& Espírito da equipe \\
& Formação de equipes e grupos \\
& Liderança \\
& Tamanho da equipe \\
\hline Processo & Arquitetura de software \\
& Engenharia de requisitos \\
& Gerência de configuração \\
& Processo de desenvolvimento \\
\hline Tecnologia & Tecnologia de colaboração \\
& Telecomunicações \\
\hline
\end{tabular}

\begin{tabular}{|l|l|}
\hline CATEGORIAS & DESAFIOS \\
\hline Gestão & Coordenação, controle e interdependência \\
& Gestão de portfólio de projetos \\
& Gerenciamento de projetos \\
& Gerência de risco \\
& Legislação (incentivos fiscais e tributários) \\
& Legislação (propriedade intelectual) \\
& Modelos de negócio \\
& Seleção e alocação de projetos \\
\hline Comunicação & Awareness \\
& Contexto \\
& Dispersão geográfica e temporal \\
& Estilo de comunicação \\
& Formas de comunicação \\
& Fusos horários \\
\hline
\end{tabular}

Fonte: Audy e Prikladnicki (2007).

\section{CENÁRIO DE ESTUDO}

No contexto de DDS, uma das subáreas da Engenharia de Software (ES), destaca-se a necessidade de experimentos para que seja provado algum determinado conceito, visto que, na área de ES, esse tipo de consciência por parte dos pesquisadores ainda é um paradigma (ZUQUELLO et al., 2013). Para isso, destaca-se a necessidade de desenvolver estudos empíricos, em especial de experimentos formais (controlados), projetos reais na indústria (estudos de caso) e surveys (por meio de entrevistas ou questionários) (AUDY; PRIKLADNICKI, 2007). 
Já Herbsleb (2005), observa que no DDS os aspectos não técnicos se tornam mais evidentes e precisam ser levados em consideração durante as pesquisas. Isto é, não devemos só investigar a parte técnica da ES, mas sim todo o contexto que o afeta, inclusive as pessoas (psicologia) e grupos (sociologia). O DDS é uma atividade de intensa interação entre membros de uma mesma equipe de trabalho.

A alocação de pessoas em ambientes de DDS é uma tarefa extremamente complexa para o gestor, pois é nesse momento que ele deve definir e distribuir as tarefas seguindo alguns critérios como, por exemplo: distância física entre os membros do projeto, facilidade de comunicação entre os membros para possíveis reuniões síncronas, afinidade sociocultural e religiosa, dentre outros fatores pessoais difíceis de mensurar e/ou classificar.

O objetivo do experimento foi avaliar o principal fator considerado pelos participantes do experimento, sobre como alocar pessoas de diferentes perfis em um mesmo projeto. Quais foram os atributos mais relevantes por elas considerados no momento dessa alocação. Para isso, responderam um questionário em que havia 36 possíveis candidatos para trabalhar com ele no projeto, com variações de perfis de forma aleatória.

A perspectiva de elaboração e análise dos dados, inclusive dos participantes é uma visão de gerente de projetos responsável pela seleção de outros membros (cinco) para participar de um determinado projeto de DDS.

O método de pesquisa utilizado foi o método empírico, onde um modelo é proposto e avaliado por meio de experimentos e/ou estudos de caso. Os estudos empíricos são tradicionalmente usados nas ciências sociais e comportamentais. Esse estudo é comum na ES, o que é fortemente determinada pelo comportamento das pessoas envolvidas no processo. Esses perfis que serão compostos por atributos considerados importantes para a seleção de pessoas para trabalhar em ambientes de DDS, conforme afirmam Audy e Prikladnicki (2007).

\section{ESTUDO EXPERIMENTAL}

A classificação do experimento foi do tipo "Estudo multi-teste para um objeto", em que há um único objeto para um conjunto de participantes. Os participantes e/ou objetos por sua vez foram selecionados aleatoriamente resultando em um experimento.

O estudo foi fundamentado nos estudos de Wohlin et al. (2000) e Perry et al. (2000) sugerindo formas de realizar experimentos controlados com pequenas alterações (OLIVEIRA JUNIOR et al., 2010). 


\subsection{Objetivo}

Os objetivos do experimento são descrito a seguir: (i) coletar dados que possam ser utilizados por gestores como fonte de informação para melhor formar suas equipes de trabalho; (ii) fornecer dados para pesquisas futuras na área; (iii) avaliar os dados coletados para extrair informações sobre a formação dos grupos de trabalho no contexto pesquisado; e (iv) contribuir para o ensino e avaliar a importância do DDS para os alunos no Curso de TI da Universidade Estadual de Maringá.

\subsection{Planejamento}

1. Seleção de Contexto: este experimento foi aplicado em domínio e acadêmico.

2. Seleção dos Participantes: estudantes de graduação e pós-graduação em Ciência da Computação, Informática e Engenharia de Produção da Universidade Estadual de Maringá (UEM).

3. Seleção de Variáveis: Como mostrado na Figura 2, as variáveis independentes são as características/perfil que os possíveis membros da equipe podem possuir: língua, estilos de comunicação, nível de formação e a dispersão geográfica; e a variável dependente é a função Rel(língua), a qual verifica se existe relação entre a língua que o participante domina com fator primordial para seleção da equipe para trabalhar em ambiente de DDS.

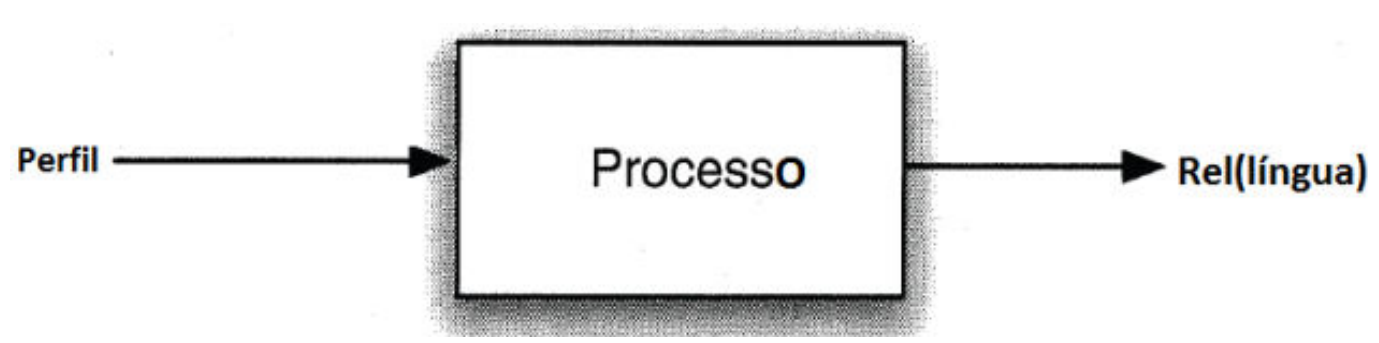

Figuras 2 - Variáveis dependentes e independentes do experimento

Fonte: Dos autores, 2013.

4. Instrumentação: o objetivo é avaliar o perfil de equipe traçado pelos participantes. Para isso, as variáveis independentes: língua, comunicação, nível de formação e 
dispersão foram medidas pelas métricas propostas. Já a variável dependente medida foi língua.

5. Formulação das Hipóteses: as seguintes hipóteses foram avaliadas no estudo:

a. Hipótese nula (H0): não existe correlação significativa das línguas, para as quais o participante possui fluência boa ou superior, com a escolha dos membros da equipe.

b. Hipótese alternativa (H1): existe correlação significativa das línguas, para as quais o participante possui fluência boa ou superior, com a escolha dos membros da equipe.

6. Experimento: todos os participantes tiveram que traçar o perfil que consideravam adequado para a formação da equipe.

\subsection{Operação}

1. Preparação: quando o experimento foi realizado todos os participantes possuíam conhecimento de Engenharia de Software e o conteúdo específico sobre DDS. O material disponibilizado para o participante do experimento foi o seguinte:

a. um teste (questionário) de caracterização, verificando os níveis de formação dos participantes, domínio de línguas e sua experiência na área de DDS;

b. um teste (questionário) para marcação dos membros(cinco desenvolvedores) que farão parte da equipe; e

c. um teste (questionário) onde o participante marcou por nível de relevância de atributos(comunicação, formação, fuso horário e língua), dos membros que farão parte da equipe.

Ao receber o questionário de caracterização, o participante teve que selecionar o nível de conhecimento das línguas que ele possui. O que foi considerado para essa caracterização foram três línguas (Português, Inglês e Espanhol), sendo que o próprio participante marca o nível de domínio sobre língua (nenhum conhecimento, fraco, razoável, bom e fluente). Ressalta-se que, para o cálculo, foram consideradas as combinações das línguas que tiveram 
marcação como "bom e fluente", formando assim mais algumas combinações (português/inglês, português/espanhol, inglês/espanhol e português/inglês/espanhol).

2. Ехесис̧ão: os participantes receberam o material descrito na Preparação (item 1). Após, foi solicitado a cada participante que fizesse a seleção dos cinco membros que fariam parte da equipe, juntamente com ele. Selecionada a equipe, deveria classificar por fator relevante, qual fator foi o principal (1 - Principal) e qual foi o alternativo (2 Alternativo) utilizado por ele para fazer a escolha dos cinco membros da equipe. Todas as tarefas foram executadas por participantes de forma individual e também sem limite de tempo para a resolução.

3. Validação dos Dados: os dados dos participantes foram coletados. Ao realizar o experimento o participante estava ciente que seus dados não seriam utilizados para outros fins a não ser de pesquisa. Também foi explicado o experimento anteriormente e solicitado ao participante que fosse o mais honesto e coerente possível nas respostas. Fazendo com que aumentasse o nível de confiabilidade do experimento. Após a realização dos testes, os mesmos foram recolhidos para posterior sumarização e avaliação dos dados.

\subsection{Análise e Interpretação}

Os dados gerados pelos participantes foram analisados e com auxílio de cálculos estatísticos e métricas foram gerados valores para a base de cálculo dos 30 resultados obtidos como demonstrado na Tabela 2. 
Tabela 2 - Valores da amostra dos participantes

\begin{tabular}{|c|c|}
\hline ID - Participante & Amostra \\
\hline 1 & 4 \\
\hline 2 & 1 \\
\hline 3 & 4 \\
\hline 4 & 4 \\
\hline 5 & 4 \\
\hline 6 & 4 \\
\hline 7 & 4 \\
\hline 8 & 1 \\
\hline 9 & 1 \\
\hline 10 & 1 \\
\hline 11 & 1 \\
\hline 12 & 1 \\
\hline 13 & 4 \\
\hline 14 & 1 \\
\hline 15 & 1 \\
\hline
\end{tabular}

\begin{tabular}{|c|c|}
\hline ID - Participante & Amostra \\
\hline 16 & 1 \\
\hline 17 & 1 \\
\hline 18 & 1 \\
\hline 19 & 4 \\
\hline 20 & 4 \\
\hline 21 & 1 \\
\hline 22 & 4 \\
\hline 23 & 4 \\
\hline 24 & 4 \\
\hline 25 & 1 \\
\hline 26 & 4 \\
\hline 27 & 4 \\
\hline 28 & 1 \\
\hline 29 & 4 \\
\hline 30 & 4 \\
\hline
\end{tabular}

Fonte: Dos autores, 2013.

1. Estatística Descritiva: a Figura 3 apresenta os valores sumarizados (Tabela 2) após análise dos resultados dos testes.

2. Normalidade dos Testes: podemos observar claramente a distribuição dos dados na Figura 4 e Figura 5 que a distribuição foi NÃO-NORMAL. Portanto para o cálculo de não normalidade utilizou-se a correlação não-paramétrica de Spearman.

3. Correlação de Spearman's: como a distribuição das amostras é não-normal, a correlação não-paramétrico de Spearman $(\rho)$ foi aplicado para apoiar a interpretação dos dados. Este método permite determinar se existe uma correlação entre os dois conjuntos de dados. Os valores de correlação são aplicados numa escala que varia entre -1,0 e 1,0, como se segue (Figura 6) -1,0 - perfeita correlação negativa, entre -1,0 e $-0,49$ - forte correlação negativa, entre $-0,5$ e $-0,01$ - correlação negativa fraca, 0 nenhuma correlação em tudo, entre 0,01 e 0,49 - correlação positiva fraca, entre 0,5 e 0,99 - forte correlação positiva, e 1.0 - correlação positiva perfeita. 
ZUQUELLO, A. G. et al. Importância dos aspectos socioculturais na gestão de equipes em ambientes de desenvolvimento distribuído de software

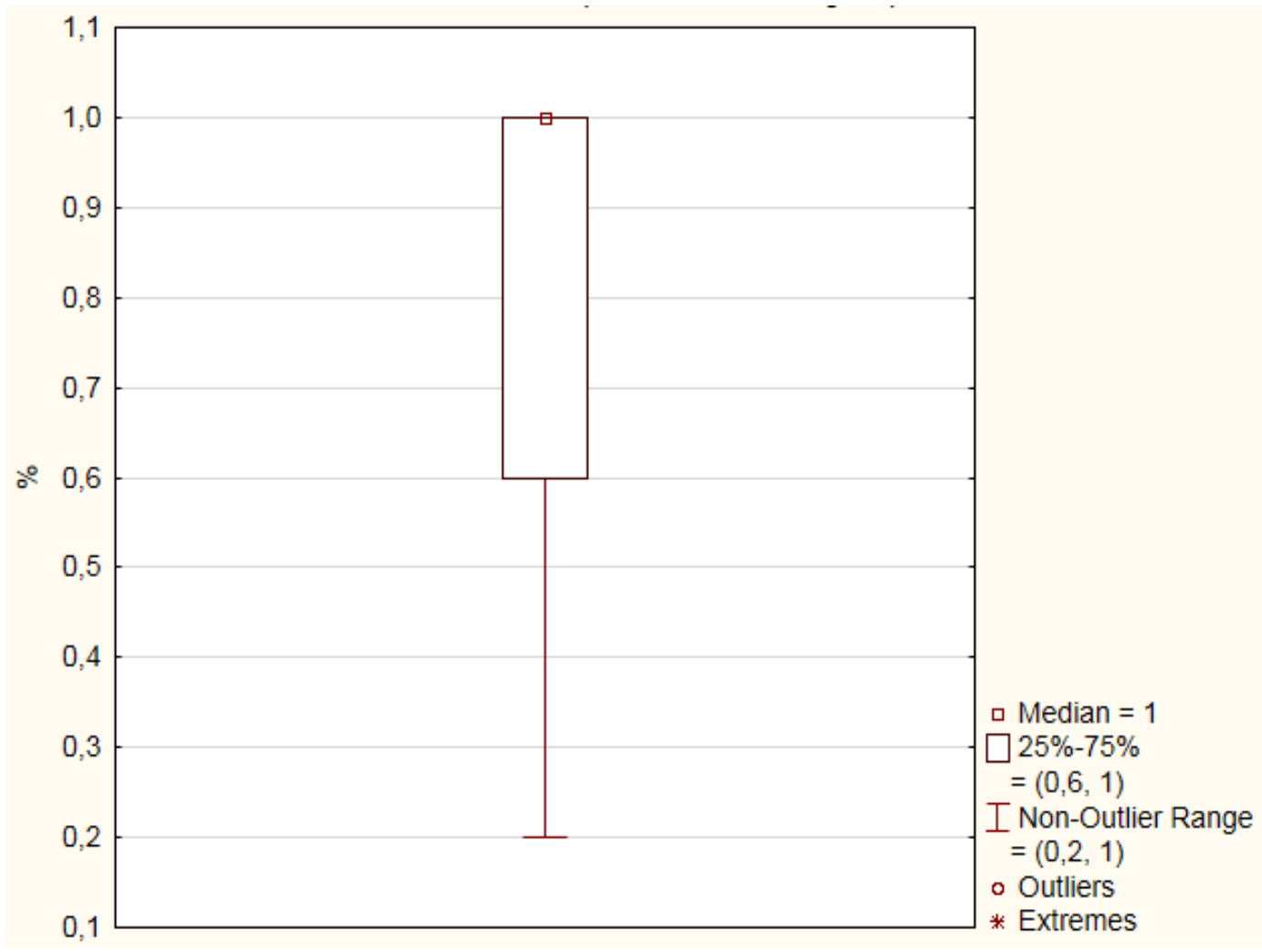

Figura 3 - Boxplot, total de pessoas que marcaram a língua que conhecem Fonte: Dos autores, 2013.

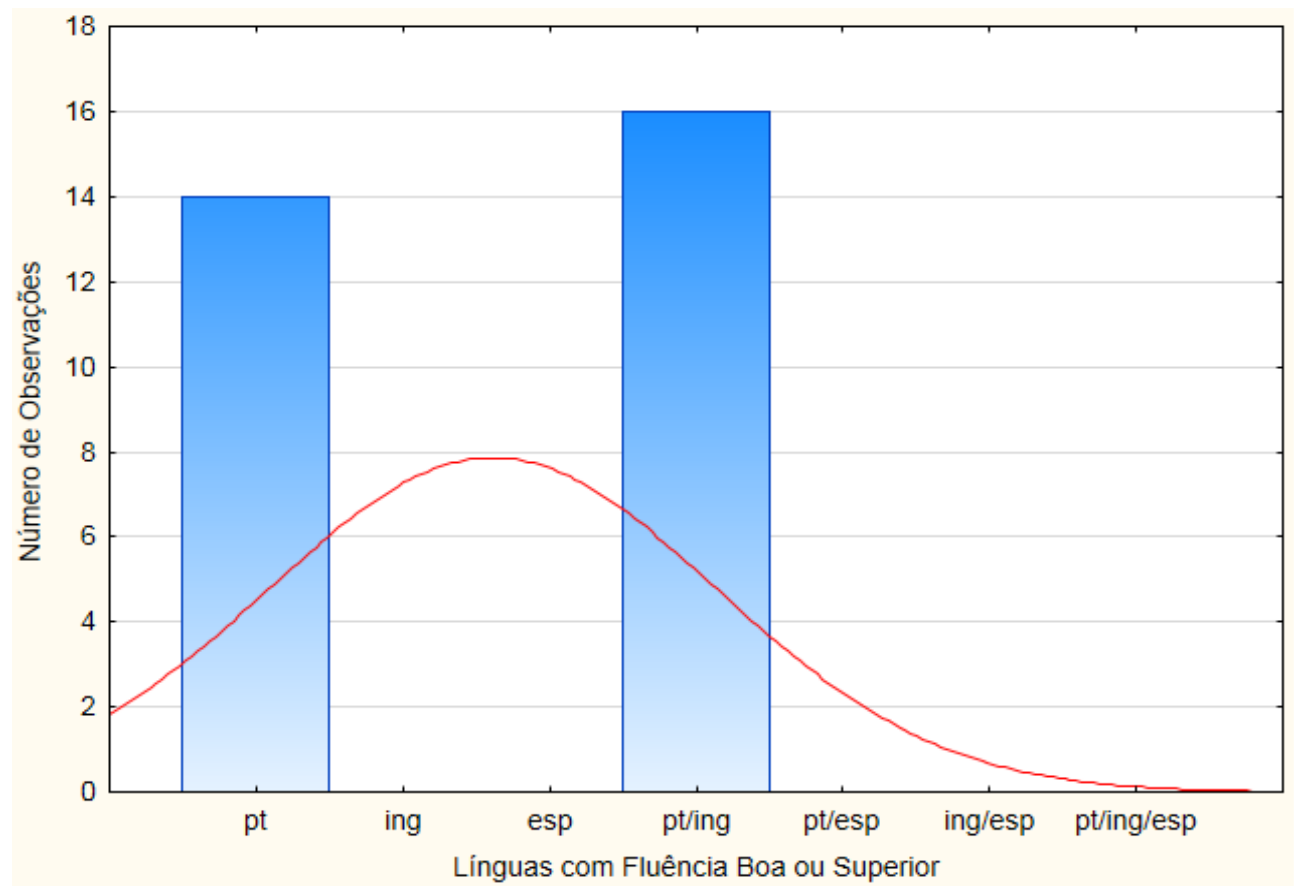

Figura 4 - Não Normalidade nos dados - Fluência Linguística Fonte: Dos autores, 2013.

ForSci.: r. cient. IFMG campus Formiga, Formiga, v. 3, n. 1, p. 01-17, jan./jun. 2015. 


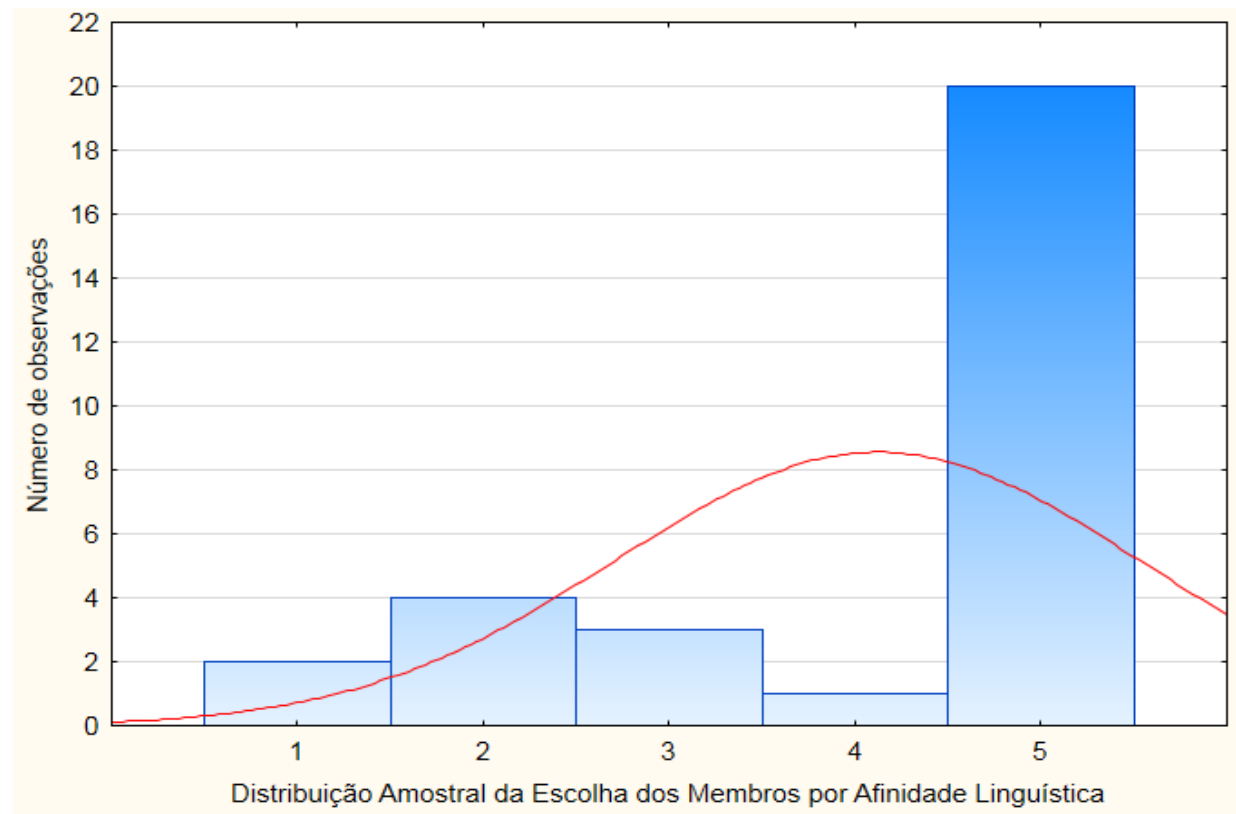

Figura 5 - Não Normalidade nos dados - Afinidade Linguística Fonte: Dos autores, 2013.

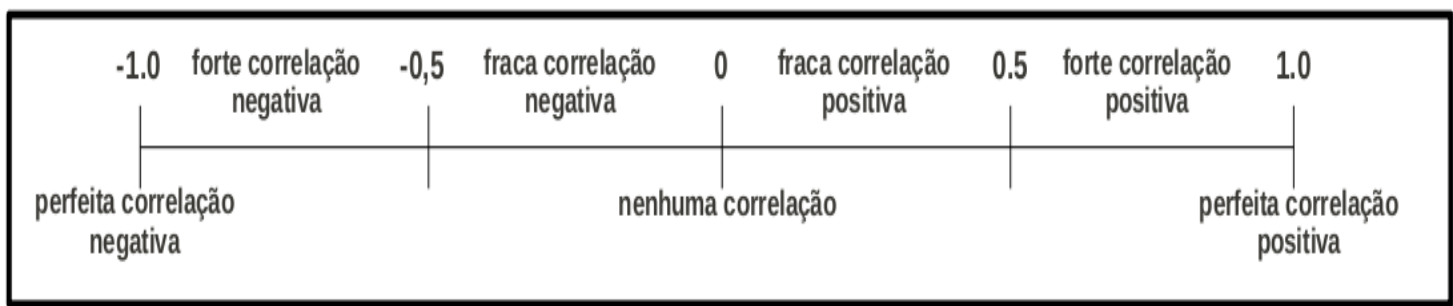

Figura 6 - Escala de Correção de Spearman

Fonte: Dos autores, 2013.

Equação 1 apresenta-se a fórmula de Spearman $(\rho)$ :

$\rho=1-\frac{6}{n\left(n^{2}-1\right)} \sum_{i=1}^{n} d_{i}^{2}$

onde: $n$ é o tamanho da amostra $(N)$

A seguinte correlação (Corr.1) foi realizada: Conjunto de línguas para os quais o participante possui fluência boa ou superior e número de membros da equipe escolhidos por saber a língua do participante, o que mostra que a língua do líder da equipe influência diretamente na sua escolha para os outros membros. Tal correlação resultou em uma forte correlação positiva $(\rho=\mathbf{0 . 8 1}\})$ (Equação 2).

$$
\left.\begin{array}{l}
\rho(\text { Corr } .1)=1-\frac{6}{30\left(30^{2}-30\right)} * 855=1-0.19= \\
0.81
\end{array}\right\}
$$


Tabela 2 - Cálculo amostral

\begin{tabular}{|c|c|c|c|c|c|c|}
\hline ID & Amostra1 & Rank1 & Amostra2 & Rank2 & D & $\mathbf{D}^{2}$ \\
\hline 1 & 4 & 8,5 & 1 & 10,5 & -2 & 4 \\
\hline 2 & 1 & 23,5 & 0,8 & 21 & 2,5 & 6,25 \\
\hline 3 & 4 & 8,5 & 1 & 10,5 & -2 & 4 \\
\hline 4 & 4 & 8,5 & 1 & 10,5 & -2 & 4 \\
\hline 5 & 4 & 8,5 & 1 & 10,5 & -2 & 4 \\
\hline 6 & 4 & 8,5 & 1 & 10,5 & -2 & 4 \\
\hline 7 & 4 & 8,5 & 1 & 10,5 & -2 & 4 \\
\hline 8 & 1 & 23,5 & 0,4 & 26,5 & -3 & 9 \\
\hline 9 & 1 & 23,5 & 0,6 & 23 & 0,5 & 0,25 \\
\hline 10 & 1 & 23,5 & 1 & 10,5 & 13 & 169 \\
\hline 11 & 1 & 23,5 & 1 & 10,5 & 13 & 169 \\
\hline 12 & 1 & 23,5 & 0,6 & 23 & 0,5 & 0,25 \\
\hline 13 & 4 & 8,5 & 1 & 10,5 & -2 & 4 \\
\hline 14 & 1 & 23,5 & 0,4 & 26,5 & -3 & 9 \\
\hline 15 & 1 & 23,5 & 0,4 & 26,5 & -3 & 9 \\
\hline
\end{tabular}

\begin{tabular}{|c|c|c|c|c|c|c|}
\hline ID & Amostra1 & Rank1 & Amostra2 & Rank2 & D & $\mathbf{D}^{2}$ \\
\hline 16 & 1 & 23,5 & 1 & 10,5 & 13 & 169 \\
\hline 17 & 1 & 23,5 & 0,2 & 29,5 & -6 & 36 \\
\hline 18 & 1 & 23,5 & 0,4 & 26,5 & -3 & 9 \\
\hline 19 & 4 & 8,5 & 1 & 10,5 & -2 & 4 \\
\hline 20 & 4 & 8,5 & 1 & 10,5 & -2 & 4 \\
\hline 21 & 1 & 23,5 & 0,2 & 29,5 & -6 & 36 \\
\hline 22 & 4 & 8,5 & 1 & 10,5 & -2 & 4 \\
\hline 23 & 4 & 8,5 & 1 & 10,5 & -2 & 4 \\
\hline 24 & 4 & 8,5 & 1 & 10,5 & -2 & 4 \\
\hline 25 & 1 & 23,5 & 0,6 & 23 & 0,5 & 0,25 \\
\hline 26 & 4 & 8,5 & 1 & 10,5 & -2 & 4 \\
\hline 27 & 4 & 8,5 & 1 & 10,5 & -2 & 4 \\
\hline 28 & 1 & 23,5 & 1 & 10,5 & 13 & 169 \\
\hline 29 & 4 & 8,5 & 1 & 10,5 & -2 & 4 \\
\hline 30 & 4 & 8,5 & 1 & 10,5 & -2 & 4 \\
\hline
\end{tabular}

Fonte: Dos autores, 2013.

\subsection{Avaliações de Validade}

Nesta seção vamos discutir sobre as ameaças à validação do estudo empírico e como tentar minimizá-las.

1. Ameaças à Validade da Conclusão: um fator que pode afetar as conclusões do experimento é a quantidade da amostra $(\mathrm{N}=30)$, no entanto, a posteriori caberá novos experimentos para corroborar para a validade das conclusões.

2. Ameaças à Validade de Construção: um fator que pode afetar na construção é o fato do experimento ter sido realizado por pessoas de uma determinada região do Brasil, fazendo com que a escolha pelo idioma português seja um fator determinante na escolha dos participantes como principal atributo de seleção. Outra língua que teve forte relevância na escolha dos participantes foi a língua inglesa, tendo em vista domínio de aplicação, acredita-se que o inglês seja a segunda língua dos participantes como é evidenciado após analisar os dados do experimento.

3. Ameaças à Validade Interna: a seguir segue algumas ameaças à validação interna:

a. Diferenças entre os participantes: um fator que pode influenciar nos resultados são as diferenças entre os participantes no quesito de experiência profissional com DDS. Os alunos de pós-graduação, possuem uma maior experiência em ambientes de DDS do que os que estão ainda cursando a graduação, pois alguns já estão trabalhando em ambientes de DDS ou áreas afins.

b. Precisão das respostas dos participantes: o comprometimento em manter uma coerência nas escolhas dos atributos pode não ter sido levado como ForSci.: r. cient. IFMG campus Formiga, Formiga, v. 3, n. 1, p. 01-17, jan./jun. 2015. 
parâmetro por alguns participantes, ocasionando incoerência nas respostas por falta de atenção na realização dos testes.

c. Outros fatores importantes: alguns fatores psicológicos dos participantes no momento da realização do experimento que não puderam ser controlados. Esses fatores não foram considerados no experimento.

\section{Ameaças à Validade Externa:}

a. Instrumentação: tentou-se contextualizar o máximo possível o experimento para que o participante estivesse o mais próximo possível da realidade.

b. Participantes: 15 participantes declaram ter um conhecimento básico sobre o contexto, isso pode gerar alguma ameaça clara ao experimento, pois podem ter respondido algumas questões de forma inconsistente.

\section{RESULTADOS E DISCUSSÕES}

Os resultados encontrados nos evidenciam que o fator linguístico, é sim o fator com maior relevância na tomada de decisão do gerente de projetos para a formação da equipe no domínio específico utilizado no experimento, o que nos leva a crer que esse é o fator com maior impacto na tomada de decisão, como ilustrado na Figura 7.

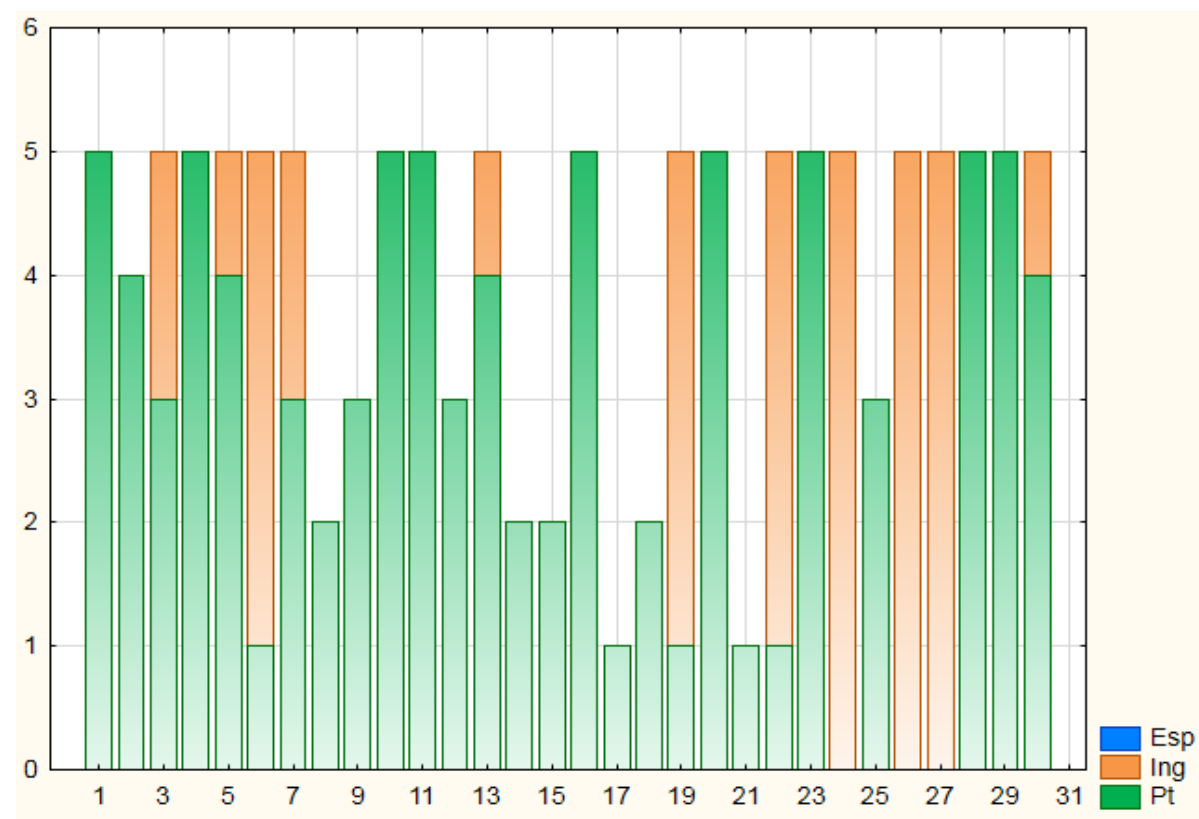

Figura 7 - Seleção de membros pela língua do participante Fonte: Dos autores, 2013.

Lembrando que o domínio que foi aplicado o experimento é um domínio acadêmico, o que se acredita, que possa divergir um pouco da realidade, caso este experimento fosse ForSci.: r. cient. IFMG campus Formiga, Formiga, v. 3, n. 1, p. 01-17, jan./jun. 2015. 
aplicado somente com pessoas que já estão atuando no mercado de trabalho e direcionados aos ambientes de DDS.

Outros experimentos e/ou replicações relacionados a esse tema devem ser feitos a posteriori e também nos mais diversos domínios para que se possa obter um maior número de evidências com relação aos fatores socioculturais na formação de equipes em ambientes de DDS.

\section{CONCLUSÃO}

Desenvolvimento de software é uma atividade colaborativa que necessita de metodologias e técnicas para resultar em sucesso. Atualmente, devido a alta demanda por experts nessas áreas, juntamente com busca pela redução de custos e prazos, vem gerando uma mudança no enfoque de organizações de desenvolvimento de software. No contexto de formação de equipes, os esforços estão sendo cada vez maiores para formar equipes coesas e que resultem em alta produtividade com uma maior redução nos custos para as organizações, melhorando assim os processos de colaboratividade em ambientes de DDS.

Neste artigo, foram analisados alguns fatores que impactam na escolha dos membros de equipes distribuídas, o foco da pesquisa foi complementar os estudos existentes sobre DDS, identificando o aspecto do indivíduo que é o mais relevante para escolha dos integrantes de um grupo de trabalho distribuído, pelo ponto de vista do líder (gestor) de uma equipe distribuída. O fator motivador para o estudo está centrado na escolha dos indivíduos e deve-se a resultados de estudos anteriores como Elonen e Artto (2003), que apresentam o recurso humano como o fator existente do desenvolvimento que mais afeta a qualidade e custo de projetos de produtos de software.

A análise de forma empírica dos dados coletados no experimento permitiu-nos evidenciar, dentro do domínio específico de realização do experimento, uma correlação forte e positiva de $\mathbf{0 , 8 1}$ pela escala de Spearman, entre o conjunto de línguas em que o líder (gestor) possui fluência e a escolha dos indivíduos com línguas correlatas ou contidas nesse conjunto.

Após a análise dos dados, foi possível identificar que dentre os fatores apresentados no estudo que a característica preponderante na tomada de decisão foi a familiaridade linguística do líder da equipe com os membros por ele escolhidos, destacando-se sobre demais elementos utilizados na investigação, como: fuso horário, comportamento e escolaridade. 
Finalmente, ressalta-se que a pesquisa foi realizada em um domínio acadêmico com todos participantes brasileiros natos. Assim sendo, existe um risco à validade do experimento, devido a cultura dos participantes, é, portanto, recomendável a replicação do experimento em um novo cenário de participantes com cultura distinta afim de corroborar com os resultados obtidos neste artigo.

\section{REFERÊNCIAS}

AUDY, J.; PRIKLADNICKI, R. Desenvolvimento distribuído de software. Rio de Janeiro: Elsevier, 2007. Disponível em: <http://books.google.com.br/books?id=znis1KYslRAC>. Acesso em: 11 nov. 2013.

BORGES, A. et al. Ontologies supporting the distributed software development: a systematic mapping study. In: INTERNATIONAL CONFERENCE ON EVALUATION AND ASSESSMENT IN SOFTWARE ENGINEERING, 17., 2013, Porto de Galinhas.

Proceedings... New York, NY: ACM, 2013. p. 153-164. Disponível em: $<$ http://doi.acm.org/10.1145/2460999.2461022>. Acesso em: 11 nov. 2013.

CARMEL, E.; TIJA, P. Offshoring information technology: sourcing and outsourcing to a global workforce. Cambridge: Cambridge University Press, 2005.

CARMEL, E. Global software teams: collaborating across borders and time zones. Upper Saddle River, NJ: Prentice Hall, 1999.

ELONEN, S.; ARTTO, K. A. Problems in managing internal development projects in multiproject environments. International Journal of Project Management, v. 21, n. 6, p. 395 402, 2003. Disponível em: <http://www.sciencedirect.com/science/article/pii/S02637863020 00972>. Acesso em: 11 nov. 2013.

HERBSLEB, J. D. Beyond computer science. In: INTERNATIONAL CONFERENCE ON SOFTWARE ENGINEERING, 27., 2005, Saint Louis, Missouri. Proceedings... New York, NY, USA: ACM, 2005. p. 23-27. Disponível em: <http://doi.acm.org/10.1145/1062455. 1062466>. Acesso em: 11 nov. 2013.

HERBSLEB, J. D. et al. E. An empirical study of global software development: distance and speed. In: INTERNATIONAL CONFERENCE ON SOFTWARE ENGINEERING, 23., 2001, Toronto, Ontario. Proceedings... Washington, DC, USA: IEEE Computer Society, 2001. p. 81-90. Disponível em: <http://dl.acm.org/citation.cfm?id=381473.381481>. Acesso em: 11 nov. 2013. 
HUZITA, E. H. M. et al. Um conjunto de soluções para apoiar o desenvolvimento distribuído de software. In: WORKSHOP DE DESENVOLVIMENTO DISTRIBUÍDO DE SOFTWARE, 2., 2008, João Pessoa. [Anais]... João Pessoa: UFPB, 2008. p. 101-110. Disponível em: <http://www.lbd.dcc.ufmg.br/colecoes/wdds/2008/011.pdf>. Acesso em: 11 nov. 2013.

HUZITA, E. H. M. et al. Um ambiente de desenvolvimento distribuído de software - DISEN. In: WORKSHOP DE DESENVOLVIMENTO DISTRIBUÍDO DE SOFTWARE, 1., 2007, João Pessoa. [Anais]... João Pessoa: UFPB, 2007. Disponível em:

$<$ http://www.lbd.dcc.ufmg.br/colecoes/wdds/2007/004.pdf >. Acesso em: 11 nov. 2013.

KAROLAK, D. W. Global software development: managing virtual teams and environments. Los Alamitos, Calif.: IEEE Computer Society, 1998.

MEYER, B. The unspoken revolution in software engineering. Computer, v. 39, n. 1, p. 124 123, Jan. 2006. Disponível em: <http://dx.doi.org/10.1109/MC.2006.37>. Acesso em: 11 nov. 2013.

OLIVEIRA JUNIOR, E. A.; MALDONADO, J. C.; GIMENES, I. M. S. Empirical validation of complexity and extensibility metrics for software product line architectures. In:

BRAZILIAN SYMPOSIUM ON SOFTWARE COMPONENTS, ARCHITECTURES AND REUSE, 4., 2010, Bahia. Proceedings... Washington, DC, USA: IEEE Computer Society, 2010. p. 31-40. Disponível em: <http://dx.doi.org/10.1109/SBCARS.2010.13>. Acesso em: 11 nov. 2013.

PAASIVAARA, M. Communication needs, practices and supporting structures in global inter-organizational software development projects. In: INTERNATIONAL WORKSHOP ON GLOBAL SOFTWARE DEVELOPMENT PROCEEDINGS, 2003, Portland, Oregon. Proceedings... Portland, Oregon: [s.n.], 2003. p. 59-63.

PRIKLADNICKI, R. MuNDDoS: um modelo de referência para desenvolvimento distribuído de software. 2003. 145 f. Dissertação (Mestrado em Ciência da Computação) Faculdade de Informática, Pontifícia Universidade Católica do Rio Grande do Sul, Porto Alegre, 2003.

PRIKLADNICKI, R. Can distributed software development help the practitioners to become better software engineers?: insights from academia. In: COMMUNITY BUILDING WORKSHOP ON COLLABORATIVE TEACHING OF GLOBALLY DISTRIBUTED SOFTWARE DEVELOPMENT, 11., 2011, Waikiki, Honolulu, HI, USA. Proceedings... New York, NY, USA: ACM, 2011. p. 16-19. Disponível em:

$<$ http://doi.acm.org/10.1145/1984665.1984669>. Acesso em: 11 nov. 2013.

PERRY, D. E.; PORTER, A. A.; VOTTA L. G. Empirical studies of software engineering: a roadmap. In: CONFERENCE ON THE FUTURE OF SOFTWARE ENGINEERING, 2000. 
Limerick, Ireland. Proceedings... New York, NY, USA: ACM, 2000. p. 345-355. Disponível em: <http://doi.acm.org/10.1145/336512.336586>. Acesso em: 11 nov. 2013.

SENGUPTA, B.; CHANDRA, S.; SINHA, V. A research agenda for distributed software development. In: INTERNATIONAL CONFERENCE ON SOFTWARE ENGINEERING, 28., 2006, Shanghai, China. Proceedings... New York, NY, USA: ACM, 2006. p. 731-740. Disponível em: <http://doi.acm.org/10.1145/1134285.1134402>. Acesso em: 11 nov. 2013.

WOHLIN, C. et al. Experimentation in software engineering: an introduction. Boston: Kluwer Academic, 2000.

ZUQUELLO, A. G. et al. A importância da experimentação na engenharia de software. In: WORKSHOP DE TECNOLOGIA DA REGIÃO FRONTEIRA OESTE, 2., 2013, Pontes e Lacerda - MT. Anais... Pontes e Lacerda: IFMT, 2013. Disponível em:

$<$ http://anaiswtrfo.plc.ifmt.edu.br/index.php/wtrfo/article/view/26/26>. Acesso em: 11 nov. 2013.

Recebido em: 22 /06/2015

Aprovado em: 28/06/2015

Publicado em: 29/06/2015 